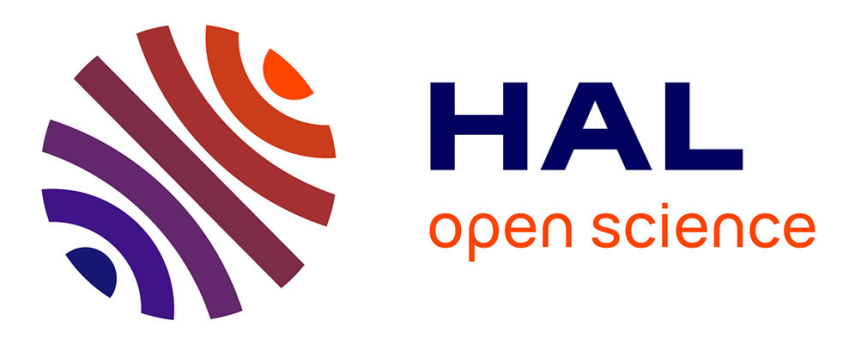

\title{
Modeling attitudes toward uncertainty through the use of the Sugeno integral
}

Alain Chateauneuf, Michel Grabisch, Agnès Rico

\section{To cite this version:}

Alain Chateauneuf, Michel Grabisch, Agnès Rico. Modeling attitudes toward uncertainty through the use of the Sugeno integral. Journal of Mathematical Economics, 2008, 44 (11), pp.1084-1099. 10.1016/j.jmateco.2007.09.003 . halshs-00327700

\section{HAL Id: halshs-00327700 \\ https://shs.hal.science/halshs-00327700}

Submitted on 9 Oct 2008

HAL is a multi-disciplinary open access archive for the deposit and dissemination of scientific research documents, whether they are published or not. The documents may come from teaching and research institutions in France or abroad, or from public or private research centers.
L'archive ouverte pluridisciplinaire HAL, est destinée au dépôt et à la diffusion de documents scientifiques de niveau recherche, publiés ou non, émanant des établissements d'enseignement et de recherche français ou étrangers, des laboratoires publics ou privés. 


\title{
Modeling attitudes toward uncertainty through the use of the Sugeno integral
}

\author{
A. Chateauneuf ${ }^{a, *}$, M. Grabisch ${ }^{\mathrm{a}}$ and A. Rico ${ }^{\mathrm{b}}$ \\ ${ }^{a}$ PSE, Centre d'Economie de la Sorbonne, Université Paris-I, Panthéon Sorbonne \\ 106-112 Bd de l'Hôpital 75647 Paris cedex 13, France \\ ${ }^{\mathrm{b}}$ LIRIS, Université Claude Bernard Lyon I \\ Bât. Braconnier, 43 Bd du 11 Novembre 1918, 69622 Villeurbanne, France
}

\begin{abstract}
The aim of this paper is to present under uncertainty, and in an ordinal framework, an axiomatic treatment of the Sugeno integral in terms of preferences which parallels some earlier derivations devoted to the Choquet integral. Some emphasis is given to the characterization of uncertainty aversion.
\end{abstract}

Key words: Sugeno integral, uncertainty aversion, preference relations.

\section{Introduction}

Under uncertainty the paradox of Ellsberg (1961) has called into question the prevailing theory of Subjective Expected Utility due to Savage (1972).

To take into account such observed behaviors, Schmeidler (1989) has built a new axiomatization of behavior. Under a key axiom, namely the comonotonic independence axiom requiring that the usual independence axiom holds only when hedging effects are absent, Schmeidler (1989) succeeded to characterize the preferences by means of a functional that turned out to be a Choquet integral (see Choquet (1953)). Moreover not only Schmeidler's model explains

* Corresponding author.

Email addresses: alain.chateauneuf@univ-paris1.fr (A. Chateauneuf), michel.grabisch@lip6.fr (M. Grabisch), agnes.rico@univ-lyon1.fr (A. Rico). 
paradoxes as Ellsberg's one, but it also offers simple and flexible representations and allows for more diversified patterns of behavior related to uncertainty aversion, by taking into account hedging effects (see e.g Schmeidler (1989), Chateauneuf (1994), and Chateauneuf and Tallon (2002)).

The so-called Choquet expected utility model presented above, as well as the classical Savage's model and most models in utility theory, are cardinal. That is, they deal with utility functions which are real-valued, and use standard operations of arithmetic like addition and multiplication.

However, it is not always easy nor desirable to deal with cardinal utility functions. A first noticing is that ordinal information is easier to get than cardinal one, and moreover, there are many situations where only order is relevant, cardinals being merely used by tradition and convenience. More fundamentally, the dominant viewpoint in economics, introduced by Hicks and Allen (1934), is that utility is related to observable choices (revealed preferences), and any introspective judgments on intensity of preference should be discarded since meaningless. As a consequence, if the only purpose of utility is to explain choices, then utility is ordinal in nature (see a discussion on ordinal vs. cardinal representations in the light of choice-based vs. choiceless utilities in Abdellaoui et al. (2002)). In other domains, as social choice theory and subjective evaluation, that is, evaluation by human subjects, ordinal representation is central. The latter typically addresses consumer opinion polls, evaluation of consumer goods (audio components, food and beverages, cosmetics) Grabisch et al. (1997), evaluation of workload and mental stress Cooper and Harper (1969); Reid and Nygren (1988), comfort Grabisch et al. (2002), etc.

It is known from measurement theory that the use of ordinal scales forbids common arithmetical operations like addition, multiplication, which require a richer scale to be meaningful (see, e.g.,Roberts (1979)). Hence, only comparisons operations are allowed, typically minimum and maximum, which we denote in the sequel by $\wedge$ and $\vee$ respectively. A key problem is then to find within this restricted toolbox, a substitute for the Choquet integral, which would allow for uncertainty representations able to cope with Ellsberg's paradox and other phenomena of uncertainty aversion. A fundamental result due to Marichal (2001) shows that more or less there is no other choice than the so-called Sugeno integral (see Sugeno (1974)). Specifically, let us consider any ordinal scale $E$ with endpoints denoted by 0 and 1 . We call Boolean polynomial any expression $P\left(x_{1}, \ldots, x_{n}\right)$ formed by variables valued in $E$, constants in $E$, linked by an arbitrary combination of parentheses, $\vee$ and $\wedge$, e.g., $\left(\left(\alpha \wedge x_{1}\right) \vee\left(x_{2} \wedge\left(\beta \vee x_{3}\right)\right)\right) \wedge x_{4}$. Then $P(0, \ldots, 0)=0$ and $P(1, \ldots, 1)=1$ if and only if $P$ is a Sugeno integral. This shows that in the ordinal case, we are forced to use the Sugeno integral. Then remains the question of studying the ability of the Sugeno integral to represent various attitude towards uncertainty, and eventually, to provide an axiomatization of what could be called a 
Sugeno expected utility model.

If the Sugeno integral has been rather well studied under a mathematical point of view (see, e.g.,Marichal (2000, 2001)), there are very few studies devoted to the use of the Sugeno integral in a decision theoretic framework (see a survey on this topic by Dubois et al. (2001a)), with a notable exception of an axiomatization à la Savage due to Dubois et al. (2001b). Our work provides an alternative axiomatization, more in the spirit of Schmeidler's work for the Choquet integral, mainly based on hedging effects, a very intuitive way of defining uncertainty aversion and uncertainty seeking. We will come up with a strong and a weak definition of uncertainty aversion (resp. seeking), providing a new characterization of necessity measures in terms of pessimism (resp., of possibility measures in terms of optimism). For conducting mathematical proofs, we will need an infinite ordinal scale, as defined in Section 2. This of course does not prevent the use of the Sugeno expected utility model on finite ordinal scales.

\section{Framework and notations}

\subsection{Algebraic and topological properties}

Let $(E, \leq)$ be a set endowed with a total order $\leq$ (a binary relation transitive, antisymmetric, and total). In addition, we assume the existence of a smallest element $0 \in E$ and a greatest element $1 \in E$. The asymmetric part of $\leq$ is denoted by $<$.

In the sequel since we only consider total orders and totally ordered sets, order and ordered will be short for total order and totally ordered.

Definition 1 Let $(E, \leq)$ and $\left(F, \leq^{\prime}\right)$ be ordered sets.

- An homomorphism $f$ of $(E, \leq)$ into $\left(F, \leq^{\prime}\right)$ is a mapping $f: E \rightarrow F$ such that

$$
x \leq y \Leftrightarrow f(x) \leq^{\prime} f(y) .
$$

- If moreover $f$ is bijective, then $f$ is an isomorphism.

Intervals of the set $E$ are defined as usually: for all $x, y \in E$,

$$
\begin{aligned}
& {[x, y]=\{z \in E \mid x \leq z \leq y\},[x, y)=\{z \in E \mid x \leq z<y\}} \\
& (x, y)=\{z \in E \mid x<z<y\},(x, y]=\{z \in E \mid x<z \leq y\} .
\end{aligned}
$$


Hence, we associate to $(E, \leq)$ a topology called order topology and denoted by $\mathcal{R}$ (see Cohen and Girard (1971)) ${ }^{1}$ :

Definition 2 The order topology, $\mathcal{R}$, on an ordered set $(E, \leq)$ is the topology generated by the intervals $(0, x)$ and $(x, 1) \forall x \in E$.

Then, $(E, \leq, \mathcal{R})$ is a topological ordered set.

In this paper, we consider an ordered set $(E, \leq)$ endowed with the order topology $\mathcal{R}$. Note that every homomorphism $f$ is continuous from $E$ to $f(E)$ according to the order topology.

We are going to give the definitions of connected and separable sets.

Definition 3 A topological ordered set $(E, \leq, \mathcal{R})$

- is connected if and only if

- it is "without gap": for all $x, y \in E$ such that $x<y$ there exists $z \in E$ which satisfies $x<z<y$;

- it is complete: each non-empty and bounded subset has a least upper bound.

- is separable if $E$ contains a countable set $A$ such that

$$
\forall x, y \in E \text { such that } x<y, \exists a \in A \text { such that } x<a<y \text {. }
$$

Such a countable set $A$ will be called an order dense subset of $E$.

To conclude we have the following result due to Eilenberg (1941):

Theorem 4 A topological separable, connected and ordered set $(E, \leq, \mathcal{R})$ is isomorphic to a real interval.

\subsection{Definitions of capacities and the Sugeno integral}

In the sequel we will assume $(E, \leq, \mathcal{R})$ totally ordered, separable, connected, and with a smallest element and a greatest element respectively denoted by 0 and 1 . We denote $E^{d}$ an order dense subset of $E$. From Theorem 4 , clearly in such a case, $(E, \leq, \mathcal{R})$ is isomorphic to the compact interval $[0,1]$.

All elements $x, y \in E$ have a least upper bound denoted $x \vee y$ and a greatest lower bound denoted $x \wedge y$.

Let $S$ be a set (of possible states of nature) endowed with a $\sigma$-algebra of events $a$ (i.e subsets of $S$ ) and $V=\{X: S \rightarrow E \mid X$ is $a$-measurable $\}$ be the set of $a$-measurable functions.

$\overline{1}$ Note that this topology exists for lattices Birkhoff (1967). 
In the sequel, we use the following standard notations:

- $\left\{X_{n}\right\}_{n \in \mathbb{N}}, X \in V$ and $X_{n} \uparrow$ C.S $X$ for simple monotone nondecreasing convergence.

- For every $A \in a, A^{*}$ denotes the characteristic function of $A$ that is $A^{*}(s)=$ 1 for all $s \in A$, and $A^{*}(s)=0$ for all $s \notin A$.

- For all $a \in E$, we denote by $a . S^{*}$ the function equal to $a$ everywhere.

To begin we give the definitions of capacities and of the Sugeno integral, in our ordinal framework.

Definition $5 A$ normalized capacity is a function $v: a \rightarrow E$ such that $v(\emptyset)=0, v(S)=1, A \subseteq B \Rightarrow v(A) \leq v(B)$.

Let $\left\{A_{n}\right\}_{n \in \mathbb{N}}$ be a sequence in $a$ and $A \in Q$ :

- $A_{n} \uparrow A$ if and only if for all $n, A_{n} \subseteq A_{n+1}$ and $\bigcup_{n \in \mathbb{N}} A_{n}=A$.

- $A_{n} \downarrow A$ if and only if for all $n, A_{n+1} \subseteq A_{n}$ and $\bigcap_{n \in \mathbb{N}} A_{n}=A$.

Definition 6 • A capacity $v$ is inner-continuous if $A_{n}, A \in A, A_{n} \uparrow A$ implies $v\left(A_{n}\right) \uparrow v(A)$.

- A capacity $v$ is outer-continuous if $A_{n}, A \in A, A_{n} \downarrow A$ implies $v\left(A_{n}\right) \downarrow$ $v(A)$.

Some particular capacities are used in this paper: the possibility measures and the necessity measures.

Definition 7 A possibility measure $\Pi$ is a normalized capacity such that:

$$
\forall A, B \in a, \quad \Pi(A \cup B)=\Pi(A) \vee \Pi(B) .
$$

Note that for $A \in a$, we have either $\Pi(A)=1$ or $\Pi(S \backslash A)=1$ or both (at least $A$ or its complement is fully possible).

Definition 8 A necessity measure (or certainty measure) $\mathcal{N}$ is a normalized capacity such that:

$$
\forall A, B \in a, \quad \mathcal{N}(A \cap B)=\mathcal{N}(A) \wedge \mathcal{N}(B)
$$

Similarly, note that for $A \in a$, we have either $\mathcal{N}(A)=0$ or $\mathcal{N}(S \backslash A)=0$ or both (at least there is no certainty on $A$ or on its complement). More on the semantics attached to possibility and necessity measures can be found in the works of Dubois and Prade (see, e.g., Dubois et al. (2000)). 
Now we give the definition of the Sugeno integral in our ordinal context as it is defined when $E=[0,1]$.

Definition 9 For all $X \in V$ and $v$ a capacity defined on $a$, the Sugeno integral of $X$ with respect to $v$ is:

$$
f X d v=\bigvee_{t \in E}(t \wedge v(X \geq t))
$$

We provide some comments and examples for illustration. Let us consider for simplicity that $S$ is a finite set, say $S=\left\{s_{1}, \ldots, s_{n}\right\}$, and take $\boldsymbol{A}=2^{S}$. Then it is easy to see that the Sugeno integral can be rewritten as follows, for any function $X: S \rightarrow E$ :

$$
f X d v=\bigvee_{i=1}^{n}\left(X\left(s_{\sigma(i)}\right) \wedge v\left(A_{\sigma(i)}\right)\right)
$$

where $\sigma$ is a permutation on $S$ so that $X$ is nondecreasing, i.e., $X\left(s_{\sigma(1)}\right) \leq$ $X\left(s_{\sigma(2)}\right) \leq \cdots \leq X\left(s_{\sigma(n)}\right)$, and $A_{\sigma(i)}=\left\{s_{\sigma(i)}, s_{\sigma(i+1)}, \ldots, s_{\sigma(n)}\right\}$.

To see how the formula works, let us consider the very simple case $S=\left\{s_{1}, s_{2}\right\}$, and consider some act $X$, assuming w.l.o.g. $X\left(s_{1}\right)<X\left(s_{2}\right)$. Then we get, since $v(S)=1$,

$$
f X d v=X\left(s_{1}\right) \vee\left(X\left(s_{2}\right) \wedge v\left(\left\{s_{2}\right\}\right)\right)=\operatorname{med}\left(X\left(s_{1}\right), X\left(s_{2}\right), v\left(\left\{s_{2}\right\}\right),\right.
$$

as it can be checked (med indicates the median of a sequence). It can be proven than the expression with the median always holds in the general (but finite) case, that is:

$$
f X d v=\operatorname{med}\left(X\left(s_{1}\right), \ldots, X\left(s_{n}\right), v\left(A_{\sigma(2)}\right), \ldots, v\left(A_{\sigma(n)}\right)\right)
$$

using the above notation. Coming back to our example with 2 states of nature, we readily see that:

- If state $s_{2}$ (that is, the state with good consequence) has a high certainty value, supposing that $X\left(s_{1}\right) \leq X\left(s_{2}\right) \leq v\left(\left\{s_{2}\right\}\right)$, then $f X d v=X\left(s_{2}\right)$. The act is evaluated like its best outcome, and the bad outcome is forgotten. Note that if $s_{1}$ has also a high certainty value (this could be the case if $v$ is a possibility measure), then the model reflects optimism.

- Conversely, if the state of good consequence has a low certainty, that is, we have $v\left(\left\{s_{2}\right\}\right) \leq X\left(s_{1}\right) \leq X\left(s_{2}\right)$, then $f X d v=X\left(s_{1}\right)$, and the act is evaluated as its worst consequence, since it is believed that $s_{1}$ will occur. Again, note that if $s_{1}$ has also a low certainty (which could be the case if $v$ is 
a certainty measure), then the model depicts pessimism. These two features (optimism with a possibility measure, pessimism with a certainty measure), have been already remarked by Dubois et al, although in different terms.

- Finally, suppose that $X\left(s_{1}\right) \leq v\left(\left\{s_{2}\right\}\right) \leq X\left(s_{2}\right)$. Then surprisingly $f X d v=$ $v\left(\left\{s_{2}\right\}\right)$, that is, the act is evaluated as the certainty of the state of good consequence. This configuration depicts a situation where the certainty of the good consequence is not enough high (compared to the utility of the outcome), so that the utility of the act is limited by the certainty of the "good" state. It may be surprising to have certainty and utility on the same scale, however this is similar to what is done in the Savage axiomatic, through Axiom S3 and binary acts, which infer a preference relation on events.

As a final illustration, let us translate the famous Savage's omelette example into our framework (this is borrowed from Dubois et al. (2001b)).

Example 10 Suppose that one wants to make a six eggs omelette, and already a 5 eggs omelette is ready. The decision problem is to know whether to add or not a 6th egg, being unsure of its freshness. Hence, we have two states of nature:

- $s_{1}$ : the egg is rotten

- $s_{2}$ : the egg is fresh.

The decision maker can choose between 3 acts:

- $X$ : break the 6th egg into the omelette

- $Y$ : break the 6th egg into a bowl, to verify its freshness

- $Z$ : throw away the egg.

The consequences are, ranked from best to worst:

- $X\left(s_{2}\right)$ : we obtain a 6 eggs omelette

- $Y\left(s_{2}\right)$ : we obtain a 6 eggs omelette, but a bowl to wash

- $Z\left(s_{1}\right)$ : we obtain a 5 eggs omelette

- $Y\left(s_{1}\right)$ : we obtain a 5 eggs omelette, plus a bowl to wash

- $Z\left(s_{2}\right)$ : we obtain a 5 eggs omelette, and an egg is wasted

- $X\left(s_{1}\right)$ : the omelette is wasted.

These consequences being ranked and supposing there is no indifference between any two of them, we may create the ordinal scale $E=\{0,1,2,3,4,5\}$, and assign each degree to the consequences according to the above order: $X\left(s_{2}\right)=5$, etc.

Let us take as model the Sugeno integral w.r.t a necessity measure $\mathcal{N}$, that is, according to our preceding analysis, a pessimistic model. Denote for simplicity 
the necessity measure of $s_{1}$ and $s_{2}$ by $n_{1}, n_{2}$ respectively. Note that necessarily $n_{1} \wedge n_{2}=0$. Using the above formula, we easily obtain:

$$
\begin{aligned}
& f X d \mathcal{N}=n_{2} \\
& f Y d \mathcal{N}=\left(n_{2} \vee 2\right) \wedge 4 \\
& f Z d \mathcal{N}=1 \text { if } n_{2}>0, \text { else }\left(n_{1} \vee 1\right) \wedge 3 .
\end{aligned}
$$

The following can be said.

- If $n_{2}=5$ (the egg is fresh for sure), then the best is to break it into the omelette $(X)$.

- If $n_{2} \in\{2,3,4\}$ (we are more or less sure that the egg is fresh), we may break it into the omelette $(X)$ or in a bowl $(Y)$.

- If $n_{2}<2$ and $n_{1}<2$ (we have very few evidence that the egg is fresh and very few evidence that the egg is rotten: state of ignorance), then we break the egg into a bowl $(Y)$.

- If $n_{1}>2$ (we think that the egg is rotten), then we throw it away (Z).

Observe how close to human intuition is the Sugeno integral model, and how it works with a very limited material.

\subsection{Characterization of the Sugeno integral}

To begin with, we need preliminary results. It is easy to check the following lemma.

Lemma 11 If $\left\{a_{n}\right\}_{n \in \mathbb{N}} \subset E$ is a nondecreasing (resp. nonincreasing) sequence in $(E, \leq, \mathcal{R})$, then $\left\{a_{n}\right\}_{n \in \mathbb{N}}$ converges in $E$ toward its least upper bound (resp. greatest lower bound).

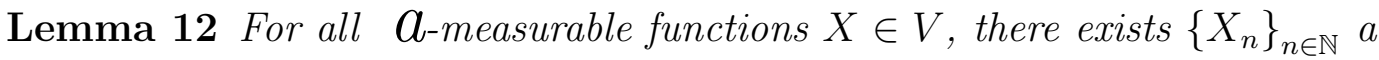
nondecreasing sequence of finite step functions which converges toward $X$.

Proof : Let $E^{d}=\left\{e_{k}\right\}_{k \in \mathbb{N}}$ be an order-dense subset of $E$. We define

$$
X_{n}=\bigvee_{k=0}^{n}\left(e_{k} \wedge\left\{X \geq e_{k}\right\}^{*}\right)
$$

$\left\{X_{n}\right\}_{n \in \mathbb{N}}$ is a nondecreasing sequence such that $X_{n} \leq X, \forall n$.

Let $s \in S$ be such that $X(s)>0$. 
For each neighborhood $V_{X(s)}$ of $X(s)$, there exists $e_{k} \in V_{X(s)} \cap E^{d}$ and $e_{k} \leq X(s)$. Then, $X_{k}$ satisfies $e_{k} \leq X_{k}(s) \leq X(s)$. Consequently, since $\left\{X_{n}(s)\right\}_{n \in \mathbb{N}}$ is nondecreasing, $\left\{X_{n}(s)\right\}_{n \in \mathbb{N}}$ converges to $X(s)$. $~$ To prove a theorem of inner-continuity of the Sugeno integral, we need a preliminary result.

\section{Lemma 13}

$$
\forall X \in V, f X d v=\bigvee_{t \in E} t \wedge v(X>t)
$$

Proof : For every $t \in E,\{X>t\} \subseteq\{X \geq t\} . v$ is a monotonic capacity so $v(X>t) \leq v(X \geq t)$ and $\bigvee_{t \in E} t \wedge v(X>t) \leq f X d v$

Let us show that $\bigvee_{t \in E} t \wedge v(X>t)<f X d v$ is impossible.

We name $m=\bigvee_{t \in E} t \wedge v(X>t)$ and $M=f X d v$ and we suppose that $m<M$.

By hypothesis, $E$ is connected so there exist $\alpha \in E$ such that $m<\alpha<M$.

$\alpha<\bigvee_{t \in E} t \wedge v(X \geq t)$, so there exists $t_{0}$ such that $\alpha<t_{0} \wedge v\left(X \geq t_{0}\right)$ which entails $\alpha<t_{0}$, so $\left\{X \geq t_{0}\right\} \subseteq\{X>\alpha\}$. $v$ is monotonic entails $v\left(X \geq t_{0}\right) \leq$ $v(X>\alpha)$ hence $\alpha<v\left(X \geq t_{0}\right)$ implies $\alpha<v(X>\alpha)$ and therefore: $\alpha \wedge$ $v(X>\alpha)=\alpha$. Consequently, $\bigvee_{t \in E} t \wedge v(X>t) \geq \alpha$ which is impossible by hypothesis.

From Lemma 13 we obtain that for the Sugeno integral, as for the Choquet integral, the integrand can be written indifferently with weak or strict inequalities.

Lemma 14 Let $X \in V,\left\{X_{n}\right\}_{n \in \mathbb{N}}$ a nondecreasing sequence in $V$ such that $X_{n} \uparrow^{C . S} X$, and $v$ be an inner-continuous capacity. Then,

$$
\lim _{n \rightarrow \infty} f X_{n} d v=f X d v
$$

Proof : From Lemma 13 we have, $f X d v=\bigvee_{t \in E} t \wedge v(X>t)$. Since for a given $t$ in $E,\left\{X_{n}>t\right\} \uparrow\{X>t\}, v$ monotone implies $v\left(X_{n}>t\right) \leq v(X>t)$, and therefore $f X_{n} d v \leq \bigvee_{t \in E}(t \wedge v(X>t))=f X d v$. $f X_{n} d v$ is a nondecreasing sequence in $E$, therefore $\lim _{n \rightarrow \infty} f X_{n} d v=\bigvee_{n} f X_{n} d v \leq f X d v$. Let $M=\bigvee_{n} f X_{n} d v$, 
hence Lemma 13 implies that for a given $t, t \wedge v\left(X_{n}>t\right) \leq M, \forall n \in \mathbb{N}$. Innercontinuity of $v$ implies $t \wedge v(X>t) \leq M$, hence $f X d v=\bigvee_{t \in E}(t \wedge v(X>t)) \leq$ $\lim _{n \rightarrow \infty} f X_{n} d v$ which completes the proof.

Note that these results remain obviously valid if $E$ is the real interval $[0,1]$.

In this paper, we focus on inner-continuity, but to better understand the Sugeno integral we study also outer-continuity. Moreover, our proofs aim at making more precise those offered by Sugeno (1974) where the capacity $v$ was (right at the beginning) assumed to be inner and outer continuous (see Sugeno's definition of fuzzy measures and of fuzzy integrals).

Lemma 15 Let $X \in V,\left\{X_{n}\right\}_{n \in \mathbb{N}}$ a nonincreasing sequence of $V$ such that $X_{n} \downarrow_{C . S} X$, and $v$ an outer-continuous capacity. Then,

$$
\lim _{n \rightarrow \infty} f X_{n} d v=f X d v
$$

Proof : Let $X \in V,\left\{X_{n}\right\}_{n \in \mathbb{N}}$ a nonincreasing sequence of $V$ such that $X_{n} \downarrow_{C . S} X$, hence $\forall n \in \mathbb{N}, \forall t \in E,\left\{X_{n} \geq t\right\} \downarrow\{X \geq t\} . v$ is monotonic, so $\forall t, v\left(X_{n} \geq t\right) \geq v(X \geq t)$. Consequently, for every $t \in E$ we have for all $n \in \mathbb{N}, t \wedge v(X \geq t) \leq t \wedge v\left(X_{n} \geq t\right) \leq \bigvee_{x \in E}\left(x \wedge v\left(X_{n} \geq x\right)\right)=f X_{n} d v$. So $f X d v \leq f X_{n} d v$, for all $n \in \mathbb{N}$, hence $f X d v \leq \bigwedge_{n \in \mathbb{N}} f X_{n} d v$.

Since $f X_{n} d v$ is a nonincreasing sequence in $E, \bigwedge_{n \in \mathbb{N}} f X_{n} d v=\lim _{n \rightarrow \infty} f X_{n} d v$ and therefore $f X d v \leq \lim _{n \rightarrow \infty} f X_{n} d v$.

Let us show that $f X d v<\bigwedge_{n \in \mathbb{N}} f X_{n} d v$ is impossible.

We name $m=f X d v, M=\bigwedge_{n \in \mathbb{N}} f X_{n} d v$ and we suppose $m<M$. $E$ is connected so there exists $\alpha \in E$ such that $m<\alpha<M$.

$\alpha<\bigwedge_{n \in \mathbb{N}} f X_{n} d v$ entails that for all $n \in \mathbb{N}, \alpha<f X_{n} d v=\bigvee_{t \in E}\left(t \wedge v\left(X_{n} \geq t\right)\right)$. Consequently, $\forall n, \exists t_{n} \in E$ such that $t_{n} \wedge v\left(X_{n} \geq t_{n}\right)>\alpha$.

We put $t^{\prime}=\bigwedge_{n} t_{n}$. For every $n, t_{n} \geq t_{n} \wedge v\left(X_{n} \geq t_{n}\right)>\alpha$ so $t^{\prime} \geq \alpha$. 
$t^{\prime} \leq t_{n}$ implies $v\left(X_{n} \geq t^{\prime}\right) \geq v\left(X_{n} \geq t_{n}\right) \geq t_{n} \wedge v\left(X_{n} \geq t_{n}\right) \geq \alpha$. Consequently, $t^{\prime} \wedge v\left(X_{n} \geq t^{\prime}\right) \geq \alpha$ for every $n$ hence $\wedge\left(t^{\prime} \wedge v\left(X_{n} \geq t^{\prime}\right)\right) \geq \alpha$. $v$ is

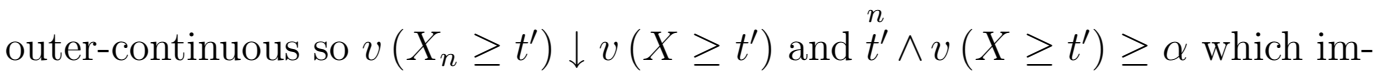
plies $f X d v \geq \alpha$. To conclude this is impossible because we have $f X d v<\alpha$.

Specific pairs of functions, called comonotonic functions, play a crucial role.

Definition $16 X, Y \in V$ are said to be comonotonic if

$$
X\left(s_{0}\right)<X\left(s_{1}\right) \Rightarrow Y\left(s_{0}\right) \leq Y\left(s_{1}\right), \forall s_{0}, s_{1} \in S .
$$

In our framework, the Sugeno integral satisfies the following usual properties proved by Denneberg and Grabisch (2004) in an ordinal scale case and Sugeno (1974) in the standard [0,1] case.

Property 1 (1) $X, X^{\prime} \in V$ comonotonic $\Rightarrow f X \vee X^{\prime} d v=f X d v \vee f X^{\prime} d v$,

(2) $X, X^{\prime} \in V$ comonotonic $\Rightarrow f X \wedge X^{\prime} d v=f X d v \wedge f X^{\prime} d v$,

(3) $v$ is a necessity measure $\Leftrightarrow \forall X, X^{\prime} \in V \quad f X \wedge X^{\prime} d v=f X d v \wedge f X^{\prime} d v$,

(4) $v$ is a possibility measure $\Leftrightarrow \forall X, X^{\prime} \in V \quad f X \vee X^{\prime} d v=f X d v \vee f X^{\prime} d v$.

\section{Proof :}

1. Let us prove that $X, X^{\prime} \in V$ comonotonic $\Rightarrow f X \vee X^{\prime} d v=f X d v \vee f X^{\prime} d v$.

Let $X, X^{\prime} \in V$ be comonotonic functions.

$f X d v \vee f X^{\prime} d v \leq f X \vee X^{\prime} d v$ since the Sugeno integral is clearly monotone with respect to the integrand i.e $X, Y \in V, X \leq Y$ implies $f X d v \leq f Y d v$.

Let us show that $f X \vee X^{\prime} d v \leq f X d v \vee f X^{\prime} d v$.

$X$ is comonotonic with $X^{\prime}$ so for every $t \in E,\{X \geq t\} \subseteq\left\{X^{\prime} \geq t\right\}$ or the converse.

- If $\{X \geq t\} \subseteq\left\{X^{\prime} \geq t\right\}$ for every $t \in E$ then from Definition 9 of the Sugeno integral $f X d v \leq f X^{\prime} d v$; note also that $\left\{X \vee X^{\prime} \geq t\right\} \subseteq\left\{X^{\prime} \geq t\right\}$ since $\left\{X \vee X^{\prime} \geq t\right\} \subseteq\{X \geq t\} \cup\left\{X^{\prime} \geq t\right\}$ hence $f X \vee X^{\prime} d v \leq f X^{\prime} d v$, therefore $f X \vee X^{\prime} d v \leq f X d v \vee f X^{\prime} d v$.

- If $\left\{X^{\prime} \geq t\right\} \subseteq\{X \geq t\}$ for every $t \in E$, a similar reasoning gives the desired result.

2. Let us prove that $X, X^{\prime} \in V$ comonotonic $\Rightarrow f X \wedge X^{\prime} d v=f X d v \wedge f X^{\prime} d v$. 
Let $X, X^{\prime} \in V$ be comonotonic functions.

$f X \wedge X^{\prime} d v \leq f X d v \wedge f X^{\prime} d v$ since the Sugeno integral is monotone. Let us show that $f X d v \wedge f X^{\prime} d v \leq f X \wedge X^{\prime} d v$. To do this we are going to prove that $f X \wedge X^{\prime} d v<f X d v \wedge f X^{\prime} d v$ is impossible.

We name $m=f X \wedge X^{\prime} d v$ and $M=f X d v \wedge f X^{\prime} d v$.

Since $E$ is connected there exists $\alpha \in E$ such that $m<\alpha<M . \alpha<$ $M$ implies that there exist $t_{0}, t_{1} \in E$ such that $\alpha<t_{0} \wedge v\left(X \geq t_{0}\right)$ and $\alpha<t_{1} \wedge v\left(X \geq t_{1}\right)$. The same reasoning as in Lemma 13 then entails: $\alpha \wedge v(X \geq \alpha)=\alpha$ and $\alpha \wedge v\left(X^{\prime} \geq \alpha\right)=\alpha . X$ is comonotonic with $X^{\prime}$ so $\{X \geq \alpha\} \subseteq\left\{X^{\prime} \geq \alpha\right\}$ or the converse.

- If $\{X \geq \alpha\} \subseteq\left\{X^{\prime} \geq \alpha\right\}$, it comes $\{X \geq \alpha\} \subseteq\left\{X \wedge X^{\prime} \geq \alpha\right\}$, and therefore $\alpha \wedge v(X \geq \alpha) \leq \alpha \wedge v\left(X \wedge X^{\prime} \geq \alpha\right)$, hence $\alpha \leq m$ a contradiction.

- The same contradiction arises if $\left\{X^{\prime} \geq \alpha\right\} \subseteq\{X \geq \alpha\}$, which completes the proof.

\section{Let us prove that}

$v$ is a necessity measure $\Leftrightarrow \forall X, X^{\prime} \in V f X \wedge X^{\prime} d v=f X d v \wedge f X^{\prime} d v$.

We consider $X, X^{\prime} \in V$ and $v$ a necessity measure.

$f X \wedge X^{\prime} d v \leq f X d v \wedge f X^{\prime} d v$ since the Sugeno integral is nondecreasing. Let us show that $f X d v \wedge f X^{\prime} d v \leq f X \wedge X^{\prime} d v$. We just have to prove that $f X \wedge X^{\prime} d v<f X d v \wedge f X^{\prime} d v$ is impossible.

We name $m=f X \wedge X^{\prime} d v$ and $M=f X d v \wedge f X^{\prime} d v$.

As previously there exists $\alpha \in E$ such that $m<\alpha<M$, and one obtains $\alpha \wedge v(X \geq \alpha)=\alpha$ and $\alpha \wedge v\left(X^{\prime} \geq \alpha\right)=\alpha$. Consequently, $\alpha=$ $\alpha \wedge\left(v(X \geq \alpha) \wedge v\left(X^{\prime} \geq \alpha\right)\right)$, but $\{X \geq \alpha\} \cap\left\{X^{\prime} \geq \alpha\right\}=\left\{X \wedge X^{\prime} \geq \alpha\right\}$. Since $v$ is a necessity measure $v(X \geq \alpha) \wedge v\left(X^{\prime} \geq \alpha\right)=v\left(X \wedge X^{\prime} \geq \alpha\right)$. Therefore $\alpha=\alpha \wedge v\left(X \wedge X^{\prime} \geq \alpha\right)$, hence $\alpha \leq m$, a contradiction which completes the proof.

The converse is immediate. Let $A, B \in a$ and let $X=A^{*}$ and $X^{\prime}=B^{*}$, the result comes immediately from $A^{*} \wedge B^{*}=(A \cap B)^{*}$.

\section{Let us prove that}

$v$ is a possibility measure $\Leftrightarrow \forall X, X^{\prime} \in V \quad f X \vee X^{\prime} d v=f X d v \vee f X^{\prime} d v$.

We consider $X, X^{\prime} \in V$ and $v$ a possibility measure.

$f X d v \vee f X^{\prime} d v \leq f X \vee X^{\prime} d v$ since the Sugeno integral is nondecreasing.

Let us prove that $f X \vee X^{\prime} d v \leq f X d v \vee f X^{\prime} d v$. 
For every $t \in E,\left\{X \vee X^{\prime} \geq t\right\}=\{X \geq t\} \cup\left\{X^{\prime} \geq t\right\}$ and $v$ is a possibility measure so $v\left(X \vee X^{\prime} \geq t\right)=v(X \geq t) \vee v\left(X^{\prime} \geq t\right)$. So for every $t \in E, t \wedge v\left(X \vee X^{\prime} \geq t\right)=t \wedge\left(v(X \geq t) \vee v\left(X^{\prime} \geq t\right)\right)=(t \wedge v(X \geq t)) \vee$ $\left(t \wedge v\left(X^{\prime} \geq t\right)\right) \leq f X d v \vee f X^{\prime} d v$. Consequently, $f X \vee X^{\prime} d v \leq f X d v \vee$ $f X^{\prime} d v$

The converse is immediate. Let $A, B \in a$ and let $X=A^{*}$ and $X^{\prime}=B^{*}$, the result comes immediately from $A^{*} \vee B^{*}=(A \cup B)^{*}$.

To conclude this section, we characterize the Sugeno integral.

Theorem 17 A functional $I: V \rightarrow E$ satisfies

(a) $I\left(a . S^{*}\right)=a, \forall a \in E$

(b) $X, Y \in V$ comonotonic $\Rightarrow I(X \vee Y)=I(X) \vee I(Y)$

(c) $X, Y \in V$ comonotonic $\Rightarrow I(X \wedge Y)=I(X) \wedge I(Y)$

(d) $X_{n} \uparrow^{C . S} X \Rightarrow I\left(X_{n}\right) \uparrow I(X)$

if and only if I is a Sugeno integral with respect to a unique inner-continuous capacity $v$.

Proof : We assume $I: V \rightarrow E$ a functional which satisfies properties (a)-(d). Let us prove that $I$ is a Sugeno integral with respect to an inner-continuous capacity.

First we consider a finite step function $X \in V$.

Let $x_{i}, i=1, \ldots, n$ be the values of the function $X$, without loss of generality, we can suppose $x_{1} \leq \ldots \leq x_{n}$, hence $X=\bigvee_{i=1}^{n} x_{i} \wedge\left\{X \geq x_{i}\right\}^{*}$.

We denote $X_{i}=x_{i} \wedge\left\{X \geq x_{i}\right\}^{*}$.

For all $i \in\{1, \ldots, n-1\}, X_{i}$ and $\bigvee_{j=i+1}^{n} X_{j}$ are comonotonic, so with (b), $I(X)=\bigvee_{i=1}^{n} I\left(x_{i} \wedge\left\{X \geq x_{i}\right\}^{*}\right)$. The function $x_{i} . S^{*}$ is constant consequently Hypotheses (c) and (a) entail $I(X)=\bigvee_{i=1}^{n} x_{i} \wedge I\left(\left\{X \geq x_{i}\right\}^{*}\right)$.

The set function $v: a \rightarrow E$ defined by $v(A)=I\left(A^{*}\right) \forall A \in Q$ is an inner-continuous capacity:

- Hypothesis (a) implies $v(\emptyset)=I(0)=0$;

- Hypothesis (a) implies $v(S)=I\left(S^{*}\right)=1$;

- Let $A \subseteq B \subseteq S$. The functions $A^{*}$ and $B^{*}$ are comonotonic such that $A^{*} \leq B^{*}$. According to (b), $I\left(B^{*}\right)=I\left(A^{*}\right) \vee I\left(B^{*}\right)$, so $I\left(A^{*}\right) \leq I\left(B^{*}\right)$. In other words, $v(A) \leq v(B)$. 
- We consider $\left\{A_{n}\right\} \subseteq a, A \in a$ such that $A_{n} \uparrow A, v\left(A_{n}\right)=I\left(A_{n}^{*}\right)$ and according to Hypothesis $(\mathbf{d}), I\left(A_{n}^{*}\right) \uparrow I\left(A^{*}\right)=v(A)$.

Consequently, $I(X)$ is a Sugeno integral of $X$ with respect to the innercontinuous capacity $v$.

\section{Let $X \in V$ be a $\boldsymbol{a}$-measurable function}

Lemma 12 implies that there exists $\left\{X_{n}\right\}_{n \in \mathbb{N}}$ a nondecreasing sequence of step functions in $V$ such that $X_{n} \uparrow^{C . S} X$. According to the first part of the proof, $I\left(X_{n}\right)=f X_{n} d v$. Hypothesis $(\mathbf{d})$ implies that $I\left(X_{n}\right)$ tends to $I(X)$ and according to Lemma $14 f X_{n} d v$ converges to $f X d v$, so $I(X)=f X d v$.

For the converse, Property 1 and Lemma 14 entail that the Sugeno integral with respect to a unique inner-continuous capacity satisfies properties (a) (d).

\section{Preferences and certainty equivalent}

We consider a decision maker (DM), who is supposed to face uncertainty (objective probabilistic information concerning the occurrence of events is not available to him) and has to choose among acts $X \in V$.

The preference relation $\preceq$ on $V$ of the (DM) is a total preorder $\preceq$ (a binary relation complete, reflexive and transitive) ${ }^{2}$.

First we state three axioms which are usual and natural requirements, whatever the attitude toward uncertainty may be.

A.1 $\succeq$ is a non-trivial total preorder,

\section{A.2 Continuity with respect to simple monotone convergence}
A.21 $\left\{a_{n}\right\}_{n \in \mathbb{N}}, a \in E, Y \in V, a_{n} \downarrow a, a_{n} . S^{*} \succeq Y \Rightarrow a . S \succeq Y$,

A.22 $\left\{X_{n}\right\}_{n \in \mathbb{N}}, X, Y \in V, Y \succeq X_{n}, X_{n} \uparrow^{C . S} X \Rightarrow Y \succeq X$

\section{A.3 Monotonicity}

A.31 $X \geq Y \Rightarrow X \succeq Y$,

A.32 $a, b \in E, a>b \Rightarrow a . S^{*} \succ b . S^{*}$.

It is straightforward to see that the previous axioms characterize the existence for every act $X$ of a certainty equivalent $I(X) \in E$, where $I: V \rightarrow E$ is monotone, monotonously continuous, and represents the preference relation $\succeq$ (we nevertheless give a proof below for sake of completeness). Namely:

$\overline{2}$ Thus for any pair of acts $X, Y, X \succeq Y$ will read $X$ is (weakly) preferred to $Y$ by the (DM); $X \succ Y$ means $X$ is strictly preferred to $Y$, and $X \sim Y$ means $X$ and $Y$ are considered as indifferent by the (DM). 
Lemma 18 A preference relation $\succeq$ on $V$ satisfies axioms $\mathbf{A} .1-\mathbf{A} .3$ if and only if for every act $X \in V$ there exists a unique element $I(X) \in E$ such that:

(i) $X \sim I(X) \cdot S^{*}$,

(ii) $X, Y \in V, X \succeq Y \Leftrightarrow I(X) \geq I(Y)$,

(iii) $X \geq Y \Rightarrow I(X) \geq I(Y)$,

(iv) $\left\{X_{n}\right\}_{n \in \mathbb{N}}, X \in V, X_{n} \uparrow^{C . S} X \Rightarrow I\left(X_{n}\right) \uparrow I(X)$.

The indifference relation $X \sim I(X) . S^{*}$ clearly justifies the denomination of $I(X)$ as the certainty equivalent of $X$.

\section{Proof :}

First we suppose that the preference relation $\succeq$ satisfies axioms A.1 - A.3.

\section{Let us prove (i)}

Let $X$ in $V$. We define $G_{X}=\left\{a \in E \mid a . S^{*} \preceq X\right\} .0 \in G_{X}$ and $\forall a \in$ $G_{X}, a \leq 1 . E$ is complete, so $G_{X}$ has a least upper bound. We name $I(X)=\sup G_{X}$. Axiom A.22 implies $I(X) . S^{*} \preceq X$, consequently, (i) follows as soon as we can show $X \preceq I(X) . S^{*}$, since the uniqueness is proved by axiom A.32.

- If $I(X)=1$, clearly $X \preceq I(X)$.S* by axiom A.31.

- If $I(X) \neq 1, E$ is separable so we can build a sequence $\left\{a_{n}\right\}_{n \in \mathbb{N}}$ in $E^{d}$ (the order dense subset of $E$ ) such that $I(X)<a_{n}<1$, $a_{n+1} \leq a_{n}, \forall n \in \mathbb{N}$ and $a_{n} \downarrow I(X)$. The elements $a_{n}$ do not below to $G_{X}$ and $\preceq$ is a total preorder so $\forall n \in \mathbb{N} a_{n} . S^{*} \succ X$. Consequently, Axiom A.21 entails $X \preceq I(X) . S^{*}$.

\section{Let us prove (ii)}

If we consider $X, Y$ in $V$ such that $X \succeq Y$, then $G_{Y} \subseteq G_{X}$ and one obtains $I(X) \leq I(Y)$.

If we consider $X, Y$ in $V$ such that $I(X) \geq I(Y)$, then Axiom A.31 entails $I(X) . S^{*} \succeq I(Y) . S^{*}$. As $I(Y) . S^{*} \sim Y$ and $I(X) . S^{*} \sim X$, the transitivity of the preference relation $\succeq$ implies that $X \succeq Y$.

\section{Let us prove (iii)}

If we consider $X, Y$ in $V$ such that $X \geq Y$, then according to Axiom A.31 we have $X \succeq Y$, hence (ii) implies $I(X) \geq I(Y)$.

\section{Let us prove (iv)}

Let $\left\{X_{n}\right\}_{n \in \mathbb{N}}$ be a sequence in $V$ such that $X_{n} \uparrow^{C . S} X$ in $V$. Then, for all $n \in \mathbb{N}$,

$$
\begin{aligned}
X_{n} \preceq X_{n+1} & \Rightarrow I\left(X_{n}\right) \leq I\left(X_{n+1}\right), \\
X_{n} \preceq X & \Rightarrow I\left(X_{n}\right) \leq I(X) .
\end{aligned}
$$

So the sequence $\left\{I\left(X_{n}\right)\right\}_{n \in \mathbb{N}}$ is a nondecreasing sequence upper bounded in $E$. According to Lemma 11, this sequence converges to its least upper bound $l$. It is easy to check, $l \leq I(X)$, let us prove $I(X) \leq l$. 
$I\left(X_{n}\right) \uparrow l$, so $X_{n} \preceq l . S^{*}$. Since $X_{n} \uparrow^{C . S} X$, Axiom A.22 entails $X \preceq$ l. $S^{*}$ which is equivalent to $I(X) \leq l$.

In conclusion we have proved that $I\left(X_{n}\right)$ converges to $I(X)$.

Now Axioms (i), (ii), (iii) and (iv) are satisfied.

Axiom A.1 is a consequence of (ii).

Let us prove A.2

Let $Y$ be in $V$ and $\left\{a_{n}\right\}_{n \in \mathbb{N}} \subseteq E$ be a sequence such that $a_{n} \downarrow a$, and $a_{n} \cdot S^{*} \succeq Y$.

If we suppose $a . S^{*} \prec Y$, then hypothesis (ii) entails $a<I(Y)$. The interval $[a, I(Y)]$ contains an open neighborhood of $a$, so there exists $N \in \mathbb{N}$ such that $a_{N}<I(Y)$. Hypothesis (ii) implies $a_{N} . S^{*} \prec Y$ which is in contradiction with the hypothesis $a_{n} . S^{*} \succeq Y, \forall n \in \mathbb{N}$. So Axiom A.21 is proved.

Let $X, Y \in V$ and $\left\{X_{n}\right\}_{n \in \mathbb{N}} \in V$ be a sequence such that $X_{n} \uparrow^{C . S} X$ and $X_{n} \preceq Y$. Properties (ii) and (iv) imply $I\left(X_{n}\right) \uparrow I(X)$ and $I\left(X_{n}\right) \leq$ $I(Y)$, so $I(X) \leq I(Y)$ and $X \preceq Y$. Then $\mathbf{A . 2 2}$ is proved.

Let us prove Axiom A.3

It is easy to check Axiom A.31 with Axioms (iii) and (ii), and finally Axiom A.32 is proved by Axioms (i) and (ii).

\section{Preferences and Sugeno integral}

We now introduce the crucial axiom A.4 of comonotonic independence which parallels a similar one for axiomatizing a simplified version of Schmeidler's model ${ }^{3}$ (see for instance Chateauneuf (1994)).

\section{A.4 Comonotonic independence}

If $X, Y, Z \in V$ are acts such that $Z$ is comonotonic with $X$ and $Y$, then:

A.41 $X \sim Y \Rightarrow X \vee Z \sim Y \vee Z$,

A.42 $X \sim Y \Rightarrow X \wedge Z \sim Y \wedge Z$.

Roughly speaking, comonotonic independence requires the direction of preference to be retained, provided hedging is not involved.

Property 2 Let $\succeq$ be a preference relation on $V$ which satisfies Axioms A.1-

\footnotetext{
$\overline{3}$ For the Schmeidler's model or else the Choquet integral, A.4.1 and A.4.2 reduce
} to a unique implication where + stands in place of $\vee($ resp. $\wedge)$. 
A.3. Then,
A.41 $\Leftrightarrow[X, Y \in V$ comonotonic $\Rightarrow I(X \vee Y)=I(X) \vee I(Y)]$,
A.42 $\Leftrightarrow[X, Y \in V$ comonotonic $\Rightarrow I(X \wedge Y)=I(X) \wedge I(Y)]$,

Proof : We suppose Axiom A.41 satisfied and we consider $X, Y \in V$ two comonotonic functions.npreuve

$X \sim I(X) . S^{*}$, and $I(X) . S^{*}$ is a constant function, so Axiom A.41 implies $X \vee Y \sim I(X) . S^{*} \vee Y$. By a similar proof, we get $I(X) . S^{*} \vee Y \sim$ $(I(X) \vee I(Y)) . S^{*}$. So $X \vee Y \sim(I(X) \vee I(Y)) . S^{*}$. The uniqueness of the certainty equivalent completes the proof.

Let us show the converse. We consider $X, Y, Z \in V$ such that $X \sim Y$ and $Z$ comonotonic with $X$ and $Y$. Hence, $I(X \vee Z)=I(X) \vee I(Z)=I(Y) \vee$ $I(Z)=I(Y \vee Z)$, in other words $X \vee Z \sim Y \vee Z$.

The proof of the second equivalence is similar.

Theorem 19 For a preference relation $\succeq$ on $V$ the following assertions are equivalent:

(i) $\succeq$ satisfies axioms A.1,A.2,A.3 and A.4

(ii) each act $X \in V$ has a certainty equivalent $I(X)$ and there exists a unique inner-continuous normalized capacity $v$ on $a$ such that

$$
\forall X \in V, I(X)=f X d v
$$

\section{Proof :}

( $i) \Rightarrow($ ii $)$ Axioms A.1, A.2, A.3 entail the existence and the uniqueness of the certainty equivalent $I$. Axiom A.4, Property 2, Lemma 18 and Theorem 17 imply that $I$ is a Sugeno integral with respect to an inner-continuous normalized capacity.

$($ ii $) \Rightarrow($ i) Theorem 17 and Lemma 18 entail the axioms A.1,A.2,A.3. Axioms A.41 and A.42 are due to (b) and (c) of Theorem 17 and Property 2 . 


\section{$5 \quad$ Uncertainty aversion and Sugeno integral}

In this section we consider $\succeq$ a preference relation on $V$ represented by a Sugeno integral. To be more precise, the preference relation $\succeq$ satisfies axioms A.1-A.4.

\subsection{Strong uncertainty aversion}

To understand axiom of strong uncertainty aversion we need a preliminary result.

Property 3 Let $X, Y, Z \in V$ be acts and $\succeq$ a preference relation on $V$ which satisfies A.1-A.4. Then,

$$
X \sim Y \text { and } Z \text { comonotonic with } Y \Rightarrow X \wedge Z \preceq Y \wedge Z \text {. }
$$

Proof : Let $X, Y, Z \in V$ be acts such that $X \sim Y$ and $Z$ comonotonic with $Y$. According to Theorem 19, the certainty equivalent is a Sugeno integral with respect to a unique inner-continuous normalized capacity. The certainty equivalent is nondecreasing so, $I(X \wedge Z) \leq I(X) \wedge I(Z)=I(Y) \wedge I(Z)$ since $X \sim Y$. Moreover, Axiom A.4 entails $I(Y) \wedge I(Z)=I(Y \wedge Z)$ so $I(X \wedge Z) \leq I(Y \wedge Z)$.

The above property entails that even if there is an asymmetric reduction of uncertainty, the (DM) can strictly prefer the more uncertain act $Y \wedge Z$ than the least uncertain $X \wedge Z$. If a decision maker, represented by a Sugeno integral, is strongly uncertainty averse, this strict preference cannot happened. Consequently, strong uncertainty aversion of a (DM) represented by a Sugeno integral is given by the following axiom:

\section{A'.4 Strong uncertainty aversion}

If $X \overline{, Y, Z \in V \text { are acts such that }} Z$ is comonotonic with $Y$, then:

$X \sim Y \Rightarrow X \wedge Z \sim Y \wedge Z$.

Lemma 20 Under Axioms A.1, A.2, A.3, A.4, Axiom A'.4 is equivalent to

$$
X \sim Y \Rightarrow X \wedge Z \sim Y \wedge Z, \forall Z \in V
$$

Proof : It is immediate that property (1) implies A'4.

Let us prove the converse. 
We consider acts $X, Y \in V$ such that $X \sim Y$, Lemma 18 yields $X \sim I(X) . S^{*}$. $Z$ is comonotonic with $I(X) . S^{*}$ so Axiom A'.4 implies $X \wedge Z \sim I(X) . S^{*} \wedge Z$. The symmetry of the hypotheses and Axiom A'.4 entail $Y \wedge Z \sim I(Y) . S^{*} \wedge Z$. $X \sim Y \Rightarrow I(X) . S^{*} \sim I(Y) . S^{*}$ and $\mathbf{A}^{\prime} .4$ implies $I(X) . S^{*} \wedge Z \sim I(Y) . S^{*} \wedge Z$. Consequently, $X \wedge Z \sim Y \wedge Z$.

Property 4 For a preference relation $\succeq$ on $V$ satisfying Axioms A.1-A.3, (i) and (ii) are equivalent:

(i) $X \sim Y \Rightarrow X \wedge Z \sim Y \wedge Z, \forall Z \in V$

(ii) $I(X \wedge Z)=I(X) \wedge I(Z), \forall X, Z \in V$.

\section{Proof :}

First we suppose $X \sim Y \Rightarrow X \wedge Z \sim Y \wedge Z, \forall Z \in V$.

$X \sim I(X) S^{*}$ entails, for all $Z \in V, X \wedge Z \sim I(X) S^{*} \wedge Z$. By a similar way, one obtains: for all $X, Z \in V, I(X) . S^{*} \wedge Z \sim(I(X) \wedge I(Z))$. $S^{*}$. So, $X \wedge Z \sim(I(X) \wedge I(Z)) . S^{*}$. The uniqueness of the certainty equivalent, achieves the proof.

Now we suppose $I(X \wedge Z)=I(X) \wedge I(Z), \forall X, Z \in V$. .

Let $X, Y$ be acts such that $X \sim Y$, hence for all $Z \in V, I(X \wedge Z)=$ $I(X) \wedge I(Z)=I(Y) \wedge I(Z)=I(Y \wedge Z)$. Consequently, $X \wedge Z \sim Y \wedge Z$.

Theorem 21 For a preference relation $\succeq$ on $V$, satisfying Axioms A.1,A.2,A.3 and A.4, (i) and (ii) are equivalent:

(i) $\succeq$ satisfies the strong uncertainty aversion axiom A'.4

(ii) There exists a unique inner-continuous necessity measure $\mathcal{N}$ on $a$ such that

$$
\forall X \in V, I(X)=f X d \mathcal{N}
$$

Proof : We have proved Theorem 19, so we just need to show that Axiom $\mathrm{A}^{\prime} .4$ is equivalent to have a necessity measure.

$(i) \Rightarrow(i i)$

Axioms A.1, A.2, A.3, A.4 entail from Theorem 19 that each act $X \in V$ has a certainty equivalent which is a Sugeno integral with respect to a unique inner-continuous normalized capacity. Adding Axiom A'.4 entails from Lemma 20 and Property 4 that $I(X \wedge Z)=I(X) \wedge I(Z), \forall X, Z \in V$. Therefore we conclude with Property 1.

$(i i) \Rightarrow(i)$ According to Property 1 , the Sugeno integral with respect to a necessity measure $\mathcal{N}$ satisfies $f X \wedge Y d \mathcal{N}=f X d \mathcal{N} \wedge f Y d \mathcal{N}, \forall X, Y \in V$. Hence Theorem 19, Property 1, Property 4 and Lemma 20 give the desired 
result.

\subsection{Weak uncertainty aversion}

A weaker form of uncertainty aversion can be obtained:

A".4 Weak uncertainty aversion

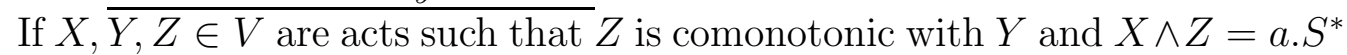
where $a \in E$ then: $X \sim Y \Rightarrow a . S^{*} \sim Y \wedge Z$.

We obtain the following characterization of weak uncertainty aversion:

Theorem 22 For a preference relation $\succeq$ on $V$, satisfying axioms A.1, A.2, A.3 and A.4, (i) and (ii) are equivalent:

(i) $\succeq$ satisfies the weak uncertainty aversion axiom $\mathbf{A}^{\prime \prime} .4$

(ii) there exists a unique inner-continuous normalized capacity $v$ on $a$ such that

$v(A) \wedge v(\bar{A})=0, \forall A \in a$, and $\forall X \in V, I(X)=f X d v$.

\section{Proof :}

$(i) \Rightarrow(i i) \quad$ Theorem 19 implies that there exists a unique inner-continuous normalized capacity $v$ on $a$ such that $\forall X \in V, I(X)=f X d v$. Let us show that $\forall A \in a, v(A) \wedge v(\bar{A})=0$.

$A^{*} \sim v(A) . S^{*}$, but $v(A) . S^{*}$ and $\bar{A}^{*}$ are comonotonic, so the property $A^{*} \wedge \bar{A}^{*}=0 . S^{*}$ and Axiom $\mathbf{A}^{\prime \prime} .4$ give $v(A) . S^{*} \wedge \bar{A}^{*} \sim 0 . S^{*}$.

We have $\bar{A}^{*} \sim v(\bar{A}) . S^{*}$. Moreover, $v(A) . S^{*}$ is comonotonic with $\bar{A}^{*}$ and $v(\bar{A}) . S^{*}$, so Axiom A.4.1 entails $\bar{A}^{*} \wedge v(A) . S^{*} \sim(v(\bar{A}) \wedge v(A)) . S^{*}$.

Consequently, $(v(\bar{A}) \wedge v(A)) . S^{*} \sim 0 . S^{*}$. The uniqueness of the certainty equivalent entails $v(A) \wedge v(\bar{A})=0$.

$(i i) \Rightarrow(i)$

Let $X, Y, Z \in V$ be such that $X \sim Y, Z$ is comonotonic with $Y$ and $X \wedge Z=a . S^{*}$. The result follows as soon as we can show that $I(Y \wedge Z)=a$.

- Let us show that $I(Y \wedge Z) \geq a$.

From Property $3, X \wedge Z \preceq Y \wedge Z$ hence $a . S^{*} \preceq Y \wedge Z$ and therefore $a \leq I(Y \wedge Z)$.

- Let us show that $I(Y \wedge Z) \leq a$.

$X \wedge Z=a . S^{*}$ implies that $\forall s \in S, X(s) \geq a$ and $Z(s) \geq a$. 
Let $A=\{s \in S \mid X(s)=a\}$, so as $X \wedge Z=a . S^{*} \bar{A}=\{s \in S \mid Z(s)=a\}$. By hypothesis, $v(A) \wedge v(\bar{A})=0$ so either $v(A)=0$ or $v(\bar{A})=0$.

- if $v(\bar{A})=0$, then $I(X)=\bigvee_{t \in E} t \wedge v(X>t) \leq a$. Consequently, $I(Y \wedge Z) \leq I(Y)=I(X) \leq a$.

- if $v(A)=0$, then $I(Z)=\bigvee_{t \in E} t \wedge v(Z>t) \leq a$. Consequently $I(Y \wedge Z) \leq I(Z) \leq a$, which completes the proof.

\subsection{Uncertainty aversion and pessimism}

In this section we argue that uncertainty aversion is intimately closely linked to pessimism. This is first illustrated by the following example (see figure 1). Acts are evaluated on an ordinal scale $E$ denoted by $\{0, a, b, c, d, e, 1\}$ with $0<a<b<c<d<e<1$. This example shows that weak uncertainty aversion expresses a kind of pessimism, and that obviously so does strong uncertainty aversion.
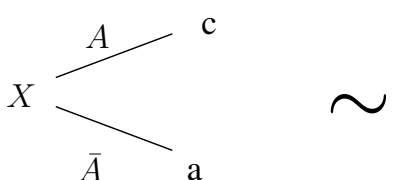

entails

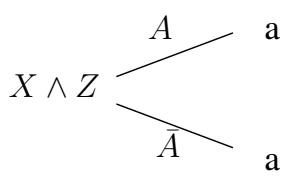

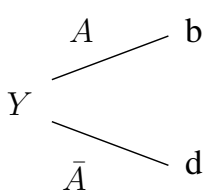

and

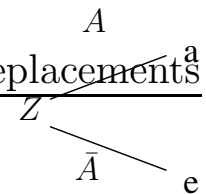

Fig. 1. Pessimism

In the above example, we consider acts $X, Y, Z$ such that $X \sim Y$ and $Y$ is comonotonic with $Z$. When we take the minimum $X \wedge Z$, uncertainty disappears. Weak uncertainty aversion axiom entails that for the decision maker (DM), $X \wedge Z \sim Y \wedge Z$. So the (DM) does not take care about $Y \wedge Z(\bar{A})=$ $d>a$, he thinks that $\bar{A}$ will not occur. Consequently, the (DM) is pessimistic.

Indeed considering the "extreme" necessity defined by $\mathcal{N}(S)=1$ and $\mathcal{N}(A)=$ 0 otherwise, clearly enlights the pessimistic state of mind of a (strongly) uncertainty averse decision maker since in such a case for any $X$ belonging to $V$, the evaluation of $X$ is given by ${ }^{4} f X d \mathcal{N}=\inf _{s \in S} X(s)$.

$\overline{4}$ Notice that it can be proved that for a connected ordered set every non-empty 


\subsection{Strong and weak uncertainty seeking}

Indeed there are symmetrical uncertainty seeking axioms.

Property 5 Let $X, Y, Z \in V$ be acts and $\succeq$ a preference relation on $V$ satisfying axioms A.1-A.4. Then,

$$
X \sim Y \text { and } Z \text { comonotonic with } Y \Rightarrow X \vee Z \succeq Y \vee Z \text {. }
$$

Proof : Let $X, Y, Z \in V$ be acts such that $X \sim Y$ and $Z$ comonotonic with $Y$. The certainty equivalent is nondecreasing so, $I(X \vee Z) \geq I(X) \vee I(Z)=$ $I(Y) \vee I(Z)$, since $X \sim Y$. Moreover, Axiom A.4 entails $I(Y) \vee I(Z)=$ $I(Y \vee Z)$

The above property entails that even if there is an asymmetric increase of uncertainty, the (DM) can strictly prefer the less uncertain act $X \vee Z$ than the more uncertain $Y \vee Z$. If a decision maker, represented by a Sugeno integral, is strongly uncertainty seeking, then this strict preference cannot happen. Consequently, strong uncertainty seeking of a (DM) represented by a Sugeno integral is given by the following axiom:

\section{B'.4 Strong uncertainty seeking}

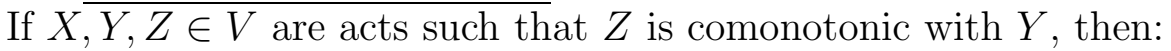
$X \sim Y \Rightarrow X \vee Z \sim Y \vee Z$.

As it has be done for the risk seeking case we can show the following result:

Lemma 23 Under axioms, A.1, A.2, A.3, A.4, Axiom $\mathbf{B}^{\prime} .4$ is equivalent to:

$$
X \sim Y \Rightarrow X \vee Z \sim Y \vee Z, \forall Z \in V
$$

A weaker form of uncertainty seeking can be obtained.

B".4 Weak uncertainty seeking

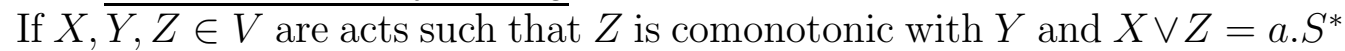
where $a \in E$ then: $X \sim Y \Rightarrow a . S^{*} \sim Y \vee Z$.

Hence we have the following results.

Theorem 24 For a preference relation $\succeq$ on $V$, satisfying Axioms A.1,A.2,A.3 and A.4, (i) and (ii) are equivalent:

bounded subset has a greatest lower bound, therefore our notation inf is consistent 
(i) $\succeq$ satisfies the strong uncertainty seeking axiom B'.4

(ii) There exists a unique inner-continuous possibility measure $\Pi$ on $a$ such that

$$
\forall X \in V, I(X)=f X d \Pi .
$$

Theorem 25 For a preference relation $\succeq$ on $V$, satisfying axioms A.1, A.2, A.3 and A.4, (i) and (ii) are equivalent:

(i) $\succeq$ satisfies the weak uncertainty aversion axiom $\mathbf{B}^{\prime \prime} .4$

(ii) there exists a unique inner-continuous normalized capacity $v$ on $a$ such that

$v(A) \vee v(\bar{A})=1, \forall A \in a$, and $\forall X \in V, I(X)=f X d v$.

To end we just illustrate optimism involved by strong uncertainty seeking through the consideration of the "extreme" possibility $\Pi$ defined by

$\Pi(A)=1, \forall A \neq \emptyset, \Pi(\emptyset)=0$. The formula $f X d \Pi=\sup _{s \in S} X(s)$ is selfexplanatory.

\section{Conclusion}

In this article, the Sugeno integral is characterized, in an ordinal framework, by axioms based on the preference relation of the decision maker. Moreover we prove that necessity measures can be characterized by a strong uncertainty aversion axiom. On the other hand capacities merely satisfying $v(A) \wedge v(\bar{A})=$ 0 for any event $A$, are shown to be related to a weaker uncertainty axiom. Finally strong and weak uncertainty seeking are dually investigated by mainly substituting the max operator to the min one.

\section{Acknowledgement}

We thank an anonymous referee for very valuable comments.

\section{References}

Abdellaoui, M., Barrios, C., Wakker, P., 2002. Reconciling introspective utility with revealed preference; experimental arguments based on prospect theory. Tech. rep., CREED, Dept. of Economics, Amsterdam, The Netherlands. 
Birkhoff, G., 1967. Lattice theory, 3rd Edition. American Mathematical Society.

Chateauneuf, A., 1994. Modeling attitudes towards uncertainty and risk through the use of Choquet integral. Annals of Operations Research 52, $3-20$.

Chateauneuf, A., Tallon, J., 2002. Diversification, convex preferences and nonempty core in the choquet expected utility model. Economic Theory 19, 509-523.

Choquet, G., 1953. Theory of capacities. Annales de l'Institut Fourier 5, 131295.

Cohen, M., Girard, B., 1971. Prordres et topologies sur un ensemble fonction d'utilit. Bulletin de Mathmatiques Economiques, universit Paris I 5.

Cooper, R. P., Harper, J., 1969. The use of pilot rating in the evaluation of aircraft handling qualities. Tech. Rep. NASA TN-D-5153, Ames Research Center, National Aeronautics and Space Administration, Moffett Field, CA.

Denneberg, D., Grabisch, M., 2004. Measure and integral with purely ordinal scales. J. of Mathematical Psychology 48, 15-27.

Dubois, D., Marichal, J.-L., Prade, H., Roubens, M., Sabbadin, R., 2001a. The use of the discrete Sugeno integral in decision making: a survey. Int. J. of Uncertainty, Fuzziness and Knowledge-Based Systems 9 (5), 539-561.

Dubois, D., Nguyen, H. T., Prade, H., 2000. Possibility theory, probability and fuzzy sets: misunderstandings, bridges and gaps. In: Dubois, D., Prade, H. (Eds.), Fundamentals of Fuzzy Sets. Vol. 1 of The Handbooks of Fuzzy Sets. Kluwer Academic Publishers, pp. 343-438.

Dubois, D., Prade, H., Sabbadin, R., 2001b. Decision-theoretic foundations of qualitative possibility theory. Eur. J. of Operational Research 128, 459-478.

Eilenberg, S., 1941. Ordered topological spaces. American Journal of Mathematics.

Ellsberg, D., 1961. Risk, ambiguity, and the Savage axioms. Quart. J. Econom. 75, 643-669.

Grabisch, M., Baret, J.-M., Larnicol, M., May 1997. Analysis of interaction between criteria by fuzzy measure and its application to cosmetics. In: Int. Conf. on Methods and Applications of Multicriteria Decision Making. Mons, Belgium, pp. 22-25.

Grabisch, M., Duchêne, J., Lino, F., Perny, P., 2002. Subjective evaluation of discomfort in sitting position. Fuzzy Optimization and Decision Making 1 (3), 287-312.

Hicks, J., Allen, A., 1934. A reconsideration of the theory of value: I, II. Economica 1, 52-75; 196-219.

Marichal, J.-L., 2000. On Sugeno integral as an aggregation function. Fuzzy Sets and Systems 114, 347-365.

Marichal, J.-L., 2001. An axiomatic approach of the discrete sugeno integral as a tool to aggregate interacting criteria in a qualitative framework. IEEE TR. on Fuzzy Systems 9 (1), 164-172.

Reid, G. B., Nygren, T. E., 1988. The subjective workload assessment tech- 
nique: A scaling procedure for measuring mental workload. In: Hancock, P. A., Meshkati, N. (Eds.), Human Mental Workload. North Holland, pp. 185-218.

Roberts, F. S., 1979. Measurement Theory. Addison-Wesley.

Savage, L. J., 1972. The Foundations of Statistics, 2nd Edition. Dover.

Schmeidler, D., 1989. Subjective probability and expected utility without additivity. Econometrica 57 (3), 571-587.

Sugeno, M., 1974. Theory of fuzzy integrals and its applications. Ph.D. thesis, Tokyo Institute of technology. 